\title{
Doceaqualip in a patient with prostate cancer who had an allergic reaction to conventional docetaxel: A case report
}

\author{
RADHESHYAM NAIK $^{1}$ and MUJTABA A. KHAN ${ }^{2}$ \\ ${ }^{1}$ Department of Medical Oncology, HCG Hospital, Bangalore, Karnataka 560027; \\ ${ }^{2}$ Department of Medical Affairs, Intas Pharmaceuticals Ltd., Ahmedabad, Gujarat 382210, India
}

Received October 7, 2016; Accepted November 29, 2016

DOI: $10.3892 / \operatorname{mco} .2017 .1147$

\begin{abstract}
Docetaxel has been widely used in the treatment of several cancers, including adenocarcinoma of the prostate gland. As docetaxel is insoluble in water, it must be administered with polysorbate 80 and ethanol, which are known to cause hypersensitivity reactions. Premedication with corticosteroids and antihistamines is advocated prior to docetaxel administration; however, toxicities, occasionally fatal, have been reported, even with corticosteroid premedication. We herein report the case of a patient with adenocarcinoma of the prostate, with bone metastasis and an Eastern Cooperative Oncology Group performance status of $<2$, who developed an allergic reaction to conventional docetaxel (Taxotere), despite being premedicated with corticosteroids and antihistamines. The patient was managed with corticosteroids and antihistamines, and was later started on Doceaqualip, a nanosomal docetaxel lipid suspension. Doceaqualip was well-tolerated by the patient and 5 cycles were subsequently administered, without any adverse reactions. Adenocarcinoma also regressed, as evidenced by positron emission tomography/computed tomography.
\end{abstract}

\section{Introduction}

Conventional taxanes, such as docetaxel, are insoluble in water. Thus, taxane formulations with surfactants such as polysorbate 80 and ethanol have been approved for the management of several cancers, including prostate adenocarcinoma (1-4). However, ethanol and polysorbate 80 have the tendency to cause infusion-related toxicities and hypersensitivity reactions (5-7). To avoid these adverse events, premedication with corticosteroids and antihistamines is advocated prior to docetaxel administration. Furthermore, several new drug

Correspondence to: Dr Radheshyam Naik, Department of Medical Oncology, HCG Hospital, 8 HCG Towers, P. Kalinga Rao Road, Sampangi, Ram Nagar, Bangalore, Karnataka 560027, India E-mail: radheshyam_n@yahoo.com

Key words: adenocarcinoma, Doceaqualip, docetaxel, nanosomal, prostate cancer delivery systems have been researched, such as liposomes, polymeric micelles, protein and nanospheres (8-14).

Nanotechnology for drug delivery has achieved significant improvements in the efficacy and safety of drugs (15-17). These nanoparticles are composed of organic substances, such as lipids, phospholipids, dextran and chitosan. The drug particles are occasionally reduced in size to nanoparticles in order to avoid use of other substances as carriers (18-20).

We herein present the case of a patient with adenocarcinoma of the prostate with bone metastasis, who developed an allergic reaction to conventional docetaxel and was later started on Doceaqualip, a nanosomal docetaxel lipid suspension (NDLS). Doceaqualip, which is devoid of polysorbate 80 and ethanol, was developed with lipids generally recognized as safe (GRAS) by the US Food and Drug Administration. Doceaqualip has achieved a higher systemic availability of docetaxel when compared with conventional docetaxel in patients with solid tumors. Furthermore, the increased efficacy of Doceaqualip compared with that of conventional docetaxel has been proven in patients with breast cancer without requirement for any premedication (18).

\section{Case report}

A 65-year-old male patient from Nairobi, Kenya, initially presented with severe back pain in August, 2015. The patient was evaluated and diagnosed with adenocarcinoma of the prostate with bone metastasis after undergoing fluorodeoxyglucose (FDG) positron emission tomography/computed tomography (PET/CT). Prostate needle biopsy revealed adenocarcinoma of the prostate with a Gleason score of $8(4+4)$ and he was treated with goserelin acetate injection and bicalutamide tablets for $\sim 3$ months. The patient had a history of hypertension that was controlled with treatment, and a history of transurethral resection of the prostate (TURP). Reassessment was performed in November, 2015. The patient had an Eastern Cooperative Oncology Group (ECOG) performance status of $<2$. A Ga-68 prostate-specific membrane antigen (PSMA) PET/CT scan revealed post-TURP changes, with small mild nodular hypermetabolism in the left posterior peripheral zone, likely representing residual prostatic disease. The PSMA revealed extensive FDG-avid heterologous osteosclerotic lesions (Fig. 1), with a standardized uptake value (SUV) of 10.5 for the prostate and 18.0 for skeletal lesions. The serum 
prostate-specific antigen (PSA) level was $0.32 \mathrm{ng} / \mathrm{ml}$. The cardiac function was normal, with an ejection fraction of $62 \%$. The blood counts were within normal limits.

Chemohormonal therapy was planned and the patient was admitted for the first cycle of chemotherapy. Injection of docetaxel $120 \mathrm{mg}$, denosumab $120 \mathrm{mg}$ and degarelix $240 \mathrm{mg}$ was scheduled, with 5 cycles planned for docetaxel. Following written informed consent, the first cycle was initiated with conventional docetaxel (Taxotere) $120 \mathrm{mg}$ after premedication with corticosteroids and antihistamines. The patient developed an anaphylactic reaction (bronchospasm, hypotension and skin rash) within $5 \mathrm{~min}$; he was administered an IV injection of chlorpheniramine maleate (Avil), hydrocortisone and paracetamol, and recovered within $10 \mathrm{~min}$. After $12 \mathrm{~h}$, the patient received $120 \mathrm{mg}$ of the NDLS Doceaqualip, which was well-tolerated without any adverse reactions. The patient subsequently received the next 3 cycles of chemotherapy with Doceaqualip. A reassessment examination performed after the third cycle of chemotherapy with a Ga-68 PSMA PET/CT scan (Fig. 1) showed mild interval regression of the small mild nodular hypermetabolism in the left posterior peripheral zone and morphologically stable heterogenous osteosclerotic lesions with internal regression of the metabolic activity. However, mild interval progression of focal hypermetabolism in the left acetabulum was present. No new metastases were observed, with an SUV of 6.9 for the prostate and 19.4 for the skeletal lesions. The patient tolerated the Doceaqualip treatment well, without any adverse events. The patient received the fifth cycle of Doceaqualip in March, 2016, along with degarelix injection $80 \mathrm{mg}$, denosumab $120 \mathrm{mg}$, filgrastim $300 \mu \mathrm{g}$ and bicalutamide tablets $50 \mathrm{mg}$. The patient was followed up 3 months later and the adenocarcinoma of the prostate had regressed considerably.

\section{Discussion}

The taxanes paclitaxel (Taxol) and docetaxel (Taxotere) have been proven to be effective as first-line therapy for metastatic cancer in several randomized clinical trials and are widely prescribed chemotherapeutic agents $(21,22)$. Docetaxel is considered to be one of the more promising taxanes, and docetaxel formulations with polysorbate 80 and ethanol (Taxotere) have been advocated in the management of different types of cancer, including prostate, non-small-cell lung, ovarian, breast and head and neck cancer. Polysorbate 80 in conventional docetaxel leaches plasticizers from polyvinylchloride infusion sets affecting the intravenously administered drug, and also causes hypersensitivity reactions, with the most common symptoms being bronchospasm, urticaria and hypotension (23). To minimize these toxicities and avoid hypersensitivity reactions, corticosteroids are used as premedication prior to the administration of docetaxel. However, corticosteroids are reported to cause hyperglycemia and increased infectious episodes (24). Fatalities due to polysorbate 80-containing docetaxel anaphylaxis may occur even after such prophylaxis (23).

Several formulations, such as taxane analogues and prodrugs, docetaxel-encapsulated nanoparticle-aptamer bioconjugates albumin nanoparticles, polyglutamates, emulsions, liposomes, docetaxel fibrinogen-coated olive oil droplets

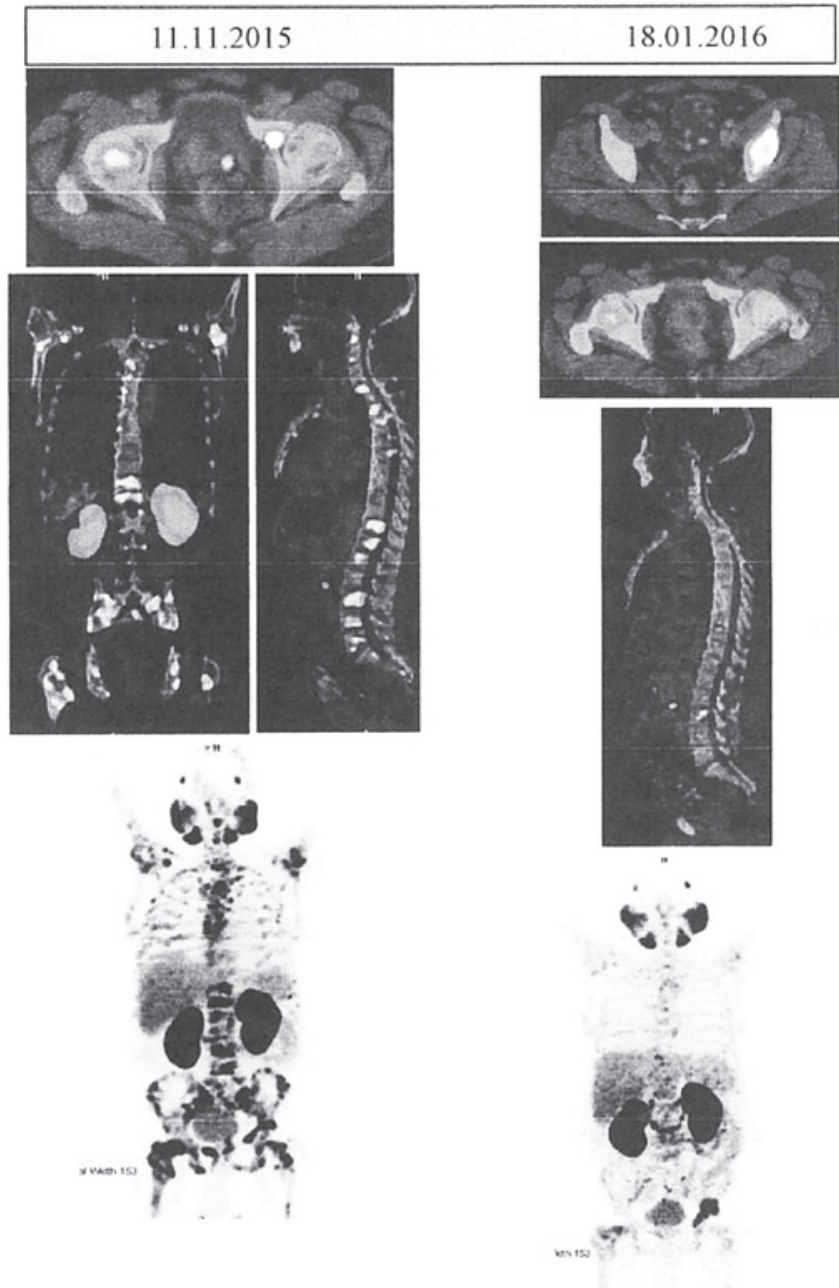

Figure 1. Comparative Ga-68 PSMA PET CT scans before and after the administration of Doceaqualip. Left panel: Images obtained in November, 2015, showing post-TURP changes, small mild nodular hypermetabolism in the left posterior peripheral zone (likely representing residual prostatic disease) and extensive FDG-avid heterologous osteosclerotic lesions. Right panel: Images obtained in January, 2016, showing post-TURP changes, mild interval regression of the nodular hypermetabolism in the left posterior peripheral zone and morphologically stable heterogenous osteosclerotic lesions with internal regression of the metabolic activity. PSMA, prostate-specific membrane antigen; PET, positron emission tomography; CT, computed tomography; TURP, transurethral resection of the prostate; FDG, fluorodeoxyglucose.

and submicronic dispersions, have been developed to avoid the abovementioned toxicities. Doceaqualip was primarily developed to avoid toxicities associated with polysorbate 80 and ethanol and to avoid premedication (18). Doceaqualip is formulated with Nano Aqualip Technology that has nano-carriers in suspension form, composed of GRAS lipids, and has demonstrated a better safety profile with a higher bioavailability compared with docetaxel (18). Moreover, even with premedication with corticosteroids and antihistamines, minor reactions (e.g., flushing and rash) occur in $\sim 40 \%$ of patients treated with conventional docetaxel $(4,18,25)$. Similarly, the patient described herein, who had adenocarcinoma of the prostate with extensive bone metastasis, developed an allergic reaction when administered conventional docetaxel with polysorbate 80 and ethanol, despite being premedicated with corticosteroids and antihistamines. The patient was immediately administered 
IV corticosteroids and antihistamines to manage the allergic reaction, and the first cycle of NDLS Doceaqualip $120 \mathrm{mg}$ $\left(75 \mathrm{mg} / \mathrm{m}^{2}\right)$ was later initiated, along with denosumab $120 \mathrm{mg}$ and filgrastim $240 \mathrm{mg}$. The treatment was well-tolerated, without any adverse reactions. The patient received further cycles without any incidents. The patient's condition also improved, as evidenced with PET scans before and after the administration of Doceaqualip (Fig. 1). In the present case, the patient developed prostate cancer post-TURP, which is consistent with the findings of Karlsson et al (26) who suggested a mildly increased risk (standardized incidence ratio: 1.26; 95\% confidence interval: $1.17-1.35)$ of prostate cancer following TURP (26).

This case report further adds to the evidence that conventional docetaxel with polysorbate 80 and ethanol has the propensity to result in adverse reactions, despite premedication with corticosteroids and antihistamines. The NDLS Doceaqualip may be an excellent tool in the management of prostate cancer with bone metastasis.

\section{Acknowledgements}

We would like to thank Dr Kamlesh Patel (Intas Pharmaceuticals Ltd.) for the necessary coordination during the development of the case report and follow-up with the journal/publisher, and Dr Venugopal Madhusudhana (Lambda Therapeutic Research) for his support in developing the concept/medical writing, additional editorial support and follow-up with the journal/publisher. Dr Mujtaba Khan is an employee of Intas Pharmaceuticals Ltd.

\section{References}

1. Valero V, Holmes FA, Walters RS, Theriault RL, Esparza L, Fraschini G, Fonseca GA, Bellet RE, Buzdar AU and Hortobagyi GN: Phase II trial of docetaxel: A new, highly effective antineoplastic agent in the management of patients with anthracycline-resistant metastatic breast cancer. J Clin Oncol 13: 2886-2894, 1995.

2. Pronk LC, Stoter G and Verweij J: Docetaxel (Taxotere): Single agent activity, development of combination treatment and reducing side-effects. Cancer Treat Rev 21: 463-478, 1995.

3. Hortobagyi GN and Kris MG: Expanding horizons: An update on the use of docetaxel in non-small cell lung, ovarian, and breast cancer. Semin Oncol 29 (3 Suppl 12): S1-S3, 2002.

4. Hennenfent KL and Govindan R: Novel formulations of taxanes: A review. Old winein a new bottle? Ann Oncol 17: 735-749, 2006.

5. Masini E, Planchenault J, Pezziardi F, Gautier P and Gagnol JP: Histamine-releasing properties of Polysorbate 80 in vitro and in vivo: Correlation with its hypotensive action in the dog. Agents Actions 16: 470-477, 1985.

6. Loos WJ, Baker SD, Verweij J, Boonstra JG and Sparreboom A: Clinical pharmacokinetics of unbound docetaxel: Role of polysorbate 80 and serum proteins. Clin Pharmacol Ther 74: 364-371, 2003.

7. Tije AJ, Verweij J, Loos WJ and Sparreboom A: Pharmacological effects of formulation vehicles: Implications for cancer chemotherapy. Clin Pharmacokinet 42: 665-685, 2003.

8. Esmaeili F, Dinarvand R, Ghahremani MH, Amini M, Rouhani H, Sepehri N, Ostad SN and Atyabi F: Docetaxel-albumin conjugates: Preparation, in vitro evaluation and biodistribution studies J Pharm Sci 98: 2718-2730, 2009.
9. Feng SS, Mei L, Anitha P, Gan CW and Zhou W: Poly (lactide)-vitamin E derivative/montmorillonite nanoparticle formulations for the oral delivery of docetaxel. Biomaterials 30 : 3297-3306, 2009.

10. Sheikh S, Ali SM, Ahmad MU, Ahmad A, Mushtaq M, Paithankar M, Mandal J, Saptarishi D, Sehgal A, Maheshwari K and Ahmad I: Nanosomal Amphotericin B is an efficacious alternative to Ambisome for fungal therapy. Int J Pharm 397: 103-108, 2010.

11. Ali SM, Ahmad A, Sheikh S, Ahmad MU, Rane RC, Kale P, Paithankar M, Saptarishi D, Sehgal A, Maheshwari K and Ahmad I: Polyoxyl 60 hydrogenated castor oil free nanosomal formulation of immunosuppressant Tacrolimus: Pharmacokinetics, safety, and tolerability in rodents and humans. Int Immunopharmacol 10: 325-330, 2010.

12. Hwang HY, Kim IS, Kwon IC and Kim YH: Tumor targetability and antitumor effect of docetaxel-loaded hydrophobically modified glycol chitosan nanoparticles. J Control Release 128: 23-31, 2008.

13. Liu B, Yang M, Li R, Ding Y, Qian X, Yu L and Jiang X: The antitumor effect of novel docetaxel-loaded thermosensitive micelles. Eur J Pharm Biopharm 69: 527-534, 2008.

14. Du W, Hong L, Yao T, Yang X, He Q, Yang B and Hu Y: Synthesis and evaluation of water-soluble docetaxel prodrugs-docetaxel esters of malic acid. Bioorg Med Chem 15: 6323-6330, 2007.

15. De Jong WH and Borm PJ: Drug delivery and nanoparticles: Applications and hazards. Int J Nanomedicine 3: 133-149, 2008.

16. Farokhzad OC and Langer R: Impact of nanotechnology on drug delivery. ACS Nano 3: 16-20, 2009.

17. Kipp JE: The role of solid nanoparticle technology in the parenteral delivery of poorly water-soluble drugs. Int J Pharm 284: 109-122, 2004.

18. Ahmad A, Sheikh S, Taran R, Srivastav SP, Prasad K, Rajappa SJ, Kumar V, Gopichand M, Paithankar M, Sharma M, et al: Therapeutic efficacy of a novel nanosomal docetaxel lipid suspension compared with taxotere in locally advanced or metastatic breast cancer patients. Clin Breast Cancer 14: 177-181, 2014.

19. De Jong WH and Borm PJ: Drug delivery and nanoparticles: Applications and hazards. Int J Nanomedicine 3: 133-149, 2008.

20. Kipp JE: The role of solid nanoparticle technology in the parenteral delivery of poorly water-soluble drugs. Int J Pharm 284: 109-122, 2004.

21. Kelly WK, Halabi S, Carducci M, George D, Mahoney JF, Stadler WM, Morris M, Kantoff P, Monk JP, Kaplan E, et al: Randomized, double-blind, placebo-controlled phase III trial comparing docetaxel and prednisone with or without bevacizumab in men with metastatic castration-resistant prostate cancer: CALGB 90401. J Clin Oncol 30: 1534-1540, 2012.

22. Urquhart LM: Taxanes as a first-line systemic treatment in metastatic breast cancer. Clin J Oncol Nurs 17 (Suppl): S15-S21, 2013.

23. Norris LB, Qureshi ZP, Bookstaver PB, Raisch DW, Sartor O, Chen H, Chen F and Bennett CL: Polysorbate 80 hypersensitivity reactions: A renewed call to action. Commun Oncol 7: 425-428, 2010.

24. Yoo KE, Kang RY, Lee JY, Lee YJ, Suh SY, Kim KS, Kim HS, Lee SH and Lee BK: Awareness of the adverse effects associated with prophylactic corticosteroid use during docetaxel therapy. Support Care Cancer 23: 1969-1977, 2015.

25. Sparano JA, Makhson AN, Semiglazov VF, Tjulandin SA, Balashova OI, Bondarenko IN, Bogdanova NV, Manikhas GM, Oliynychenko GP, Chatikhine VA, et al: Pegylated liposomal doxorubicin plus docetaxel significantly improves time to progression without additive cardiotoxicity compared with docetaxel monotherapy in patients with advanced breast cancer previously treated with neoadjuvant-adjuvant anthracycline therapy: Results from a randomized phase III study. J Clin Oncol 27: 4522-4529, 2009.

26. Karlsson CT, Wiklund F, Grönberg H, Bergh A and Melin B: Risk of prostate cancer after trans urethral resection of $\mathrm{BPH}$ : A cohort and nested case-control study. Cancers (Basel) 3: 4127-4138, 2011. 